\title{
Two- and three-gluon glueballs of $C=+$
}

\author{
Hua-Xing Chen $\oplus^{1,{ }^{*}}$ Wei Chen $\oplus^{2, \dagger}$ and Shi-Lin Zhu $\oplus^{3, *}$ \\ ${ }^{1}$ School of Physics, Southeast University, Nanjing 210094, China \\ ${ }^{2}$ School of Physics, Sun Yat-Sen University, Guangzhou 510275, China \\ ${ }^{3}$ School of Physics and Center of High Energy Physics, Peking University, Beijing 100871, China
}

(Received 28 July 2021; accepted 5 November 2021; published 30 November 2021)

\begin{abstract}
We study two- and three-gluon glueballs of $C=+$ using the method of QCD sum rules. We systematically construct their interpolating currents, and find that all the spin- 1 currents of $C=+$ vanish. This suggests that the "ground-state" spin-1 glueballs of $C=+$ do not exist within the relativistic framework. We calculate masses of the two-gluon glueballs with $J^{P C}=0^{ \pm+} / 2^{ \pm+}$and the three-gluon glueballs with $J^{P C}=0^{ \pm+} / 2^{ \pm+}$. We propose searching for the $J^{P C}=0^{-+} / 2^{- \pm} / 3^{ \pm-}$three-gluon glueballs in their three-meson decay channels in the future BESIII, GlueX, LHC, and PANDA experiments.
\end{abstract}

DOI: 10.1103/PhysRevD.104.094050

\section{INTRODUCTION}

Glueballs, composed of valence gluons, are important for the understanding of nonperturbative QCD [1-3]. There have been tremendous theoretical studies on them in the past fifty years using various models and methods, such as the MIT bag model [4], the flux-tube model [5], the Coulomb Gauge model [6,7], Regge trajectories [8], holographic QCD [9], lattice QCD [10-14], and QCD sum rules [15-30], etc. However, experimental efforts in searching for glueballs are confronted with the difficulty of identifying them unambiguously, and there is currently no definite experimental evidence for their existence.

Recently the D0 and TOTEM Collaborations studied $p p$ and $p \bar{p}$ [31] cross sections, which are found to be different with a significance of $3.4 \sigma$ [32]. Together with their previous result [33], this significance can be increased to $5.2 \sigma-5.7 \sigma$. The above difference leads to the evidence of a $t$-channel exchanged odderon [34-38], that is predominantly a threegluon glueball of $C=-$. We refer to Refs. [39-47] and a review of [48] for more discussions. Due to these studies, interest in glueballs have recently been revived. Since the above odderon evidence is still indirect, it is crucial and important to directly study the glueball itself.

The lowest-lying two-, three-, and four-gluon glueballs have been systematically investigated in Ref. [49], where the

\footnotetext{
*hxchen@seu.edu.cn

†chenwei29@mail.sysu.edu.cn

zhusl@pku.edu.cn
}

Published by the American Physical Society under the terms of the Creative Commons Attribution 4.0 International license. Further distribution of this work must maintain attribution to the author(s) and the published article's title, journal citation, and DOI. Funded by SCOAP ${ }^{3}$. authors constructed their corresponding nonrelativistic lowdimension operators. These operators have been successfully used in lattice QCD calculations. In this paper we systematically study two- and three-gluon glueballs of $C=+$. We shall construct their corresponding relativistic glueball currents, and calculate the masses of these glueballs using the method of QCD sum rules. The same approach has been applied in Ref. [50] to study three-gluon glueballs of $C=-$, so a rather complete $\mathrm{QCD}$ sum rule study will be done on the lowest-lying glueballs composed of two- or three-valence gluons. These studies can largely improve our understanding of the gluon degree of freedom as well as the nonperturbative behaviors of the strong interaction at the low-energy region.

This paper is organized as follows. We systematically construct relativistic two- and three-gluon glueball currents of $C=+$ in Sec. II. We apply them to perform QCD sum rule analyses in Sec. III, and perform numerical analyses in Sec. IV. The obtained results are summarized and discussed in Sec. V, and are compared with lattice QCD results [11-14].

\section{RELATIVISTIC GLUEBALL CURRENTS}

In this section we systematically construct relativistic glueball currents, including the two-gluon glueball currents and the $C=+$ three-gluon glueball currents. We shall do this separately in the following subsections. Note that the two-gluon glueball currents cannot reach $C=-$ [51], and the $C=-$ three-gluon glueball currents have been systematically constructed in Ref. [50].

\section{A. Couplings of tensor currents}

In the present study we shall use some special tensor currents to study glueballs with nonzero spins $J \neq 0$. These currents have $2 \times J$ Lorentz indices with certain symmetries, 
and they couple to both positive- and negative-parity glueballs. In this subsection we briefly explain how we deal with them.

We assume $J_{\alpha \beta}$ to be a tensor current with two antisymmetric Lorentz indices $\mu$ and $\nu$. Taking the current $J_{\alpha \beta}=\bar{c} \sigma_{\alpha \beta} c$ as an example, it can be separated into $(\alpha, \beta=0,1,2,3$ and $i, j=1,2,3)$

$$
J_{\alpha \beta}=\bar{c} \sigma_{\alpha \beta} c \rightarrow \begin{cases}\bar{c} \sigma_{i j} c, & P=+ \\ \bar{c} \sigma_{0 i} c, & P=-.\end{cases}
$$

Accordingly, it couples to both positive- and negativeparity charmonia through

$$
\begin{gathered}
\left\langle 0\left|J_{\alpha \beta}\right| h_{c}(\epsilon, p)\right\rangle=i f_{h_{c}}^{T} \epsilon_{\alpha \beta \mu \nu} \epsilon^{\mu} p^{\nu}, \\
\left\langle 0\left|J_{\alpha \beta}\right| J / \psi(\epsilon, p)\right\rangle=i f_{J / \psi}^{T}\left(p_{\alpha} \epsilon_{\beta}-p_{\beta} \epsilon_{\alpha}\right),
\end{gathered}
$$

where $f_{h_{c}}^{T}$ and $f_{J / \psi}^{T}$ are relevant decay constants. Given the Lorentz structures of $J / \psi$ and $h_{c}$ are totally different, they can be clearly separated from each other. For example, we can isolate $h_{c}$ at the hadron level by investigating the twopoint correlation function containing

$$
\begin{aligned}
& \left\langle 0\left|J_{\alpha \beta}\right| h_{c}\right\rangle\left\langle h_{c}\left|J_{\alpha^{\prime} \beta^{\prime}}^{\dagger}\right| 0\right\rangle \\
& =\left(f_{h_{c}}^{T}\right)^{2} \epsilon_{\alpha \beta \mu \nu} \epsilon^{\mu} p^{\nu} \epsilon_{\alpha^{\prime} \beta^{\prime} \mu^{\prime} \nu^{\prime}} \epsilon^{* \mu^{\prime}} p^{\nu^{\prime}} \\
& =-\left(f_{h_{c}}^{T}\right)^{2} p^{2}\left(g_{\alpha \alpha^{\prime}} g_{\beta \beta^{\prime}}-g_{\alpha \beta^{\prime}} g_{\beta \alpha^{\prime}}\right)+\cdots,
\end{aligned}
$$

since the correlation function of $J / \psi$ does not contain the above coefficient. It is not so easy to isolate $J / \psi$ from $J_{\alpha \beta}$ at the hadron level. Instead, we can investigate its partner current

$$
\tilde{J}_{\alpha \beta}=\epsilon_{\alpha \beta \gamma \delta} \times J^{\gamma \delta},
$$

which couples to $J / \psi$ and $h_{c}$ (just in the opposite way)

$$
\begin{aligned}
\left\langle 0\left|\tilde{J}_{\alpha \beta}\right| J / \psi(\epsilon, p)\right\rangle & =i \tilde{f}_{J / \psi}^{T} \epsilon_{\alpha \beta \mu \nu} \epsilon^{\mu} p^{\nu}, \\
\left\langle 0\left|\tilde{J}_{\alpha \beta}\right| h_{c}(\epsilon, p)\right\rangle & =i \tilde{f}_{h_{c}}^{T}\left(p_{\alpha} \epsilon_{\beta}-p_{\beta} \epsilon_{\alpha}\right) .
\end{aligned}
$$

Accordingly, we can use the two currents $J_{\alpha \beta}$ and $\tilde{J}_{\alpha \beta}$ to study and separate $J / \psi$ and $h_{c}$.

We apply the above process to generally investigate the current $J^{\alpha_{1} \cdots \alpha_{N}, \beta_{1} \cdots \beta_{N}}$, which has $2 N=2 J$ Lorentz indices with certain symmetries, e.g., the spin-2 current $J^{\alpha_{1} \alpha_{2}, \beta_{1} \beta_{2}}$ has four Lorentz indices, satisfying

$$
J^{\alpha_{1} \alpha_{2}, \beta_{1} \beta_{2}}=-J^{\beta_{1} \alpha_{2}, \alpha_{1} \beta_{2}}=-J^{\alpha_{1} \beta_{2}, \beta_{1} \alpha_{2}}=J^{\alpha_{2} \alpha_{1}, \beta_{2} \beta_{1}} .
$$

Its coupling can be written as

$$
\left\langle 0\left|J^{\alpha_{1} \cdots \alpha_{N}, \beta_{1} \cdots \beta_{N}}\right| X\right\rangle=i f_{X} \mathcal{S}\left[\epsilon^{\alpha_{i} \beta_{i} \mu_{i} \nu_{i}} p_{\nu_{i}}\right]^{N} \epsilon_{\mu_{1} \cdots \mu_{N}},
$$

where $X$ is the corresponding state having the same parity as $J^{i_{1} \cdots i_{N}, j_{1} \cdots j_{N}}\left(i_{1} \cdots j_{N}=1,2,3\right) ; \mathcal{S}$ denotes symmetrization and subtracting trace terms in the two sets $\left\{\alpha_{1} \cdots \alpha_{N}\right\}$ and $\left\{\beta_{1} \cdots \beta_{N}\right\}$ simultaneously, with

$$
[\cdots]^{N}=\epsilon^{\alpha_{1} \beta_{1} \mu_{1} \nu_{1}} p_{\nu_{1}} \cdots \epsilon^{\alpha_{N} \beta_{N} \mu_{N} \nu_{N}} p_{\nu_{N}} .
$$

Note that the current $J^{\alpha_{1} \cdots \alpha_{N}, \beta_{1} \cdots \beta_{N}}$ can also couple to the other state $X^{\prime}$ having opposite parity to $X$, but this state $X^{\prime}$ cannot be easily isolated at the hadron level, so we do not consider it in the present study.

\section{B. Two-gluon glueball currents}

In this subsection we use the gluon field strength tensor $G_{\mu \nu}^{a}$ to construct two-gluon glueball currents, with $a$ the color index and $\mu, \nu$ the Lorentz indices. We also need $\tilde{G}_{\mu \nu}^{a}=G^{a, \rho \sigma} \times \epsilon_{\mu \nu \rho \sigma} / 2$ to denote the dual gluon-field strength tensor, and $f^{a b c}$ to denote the totally antisymmetric $S U(3)_{C}$ structure constant. In the present study we only consider local glueball currents without explicit derivatives, although $G_{\mu \nu}^{a}$ and $\tilde{G}_{\mu \nu}^{a}$ contain covariant derivatives within them.

In Ref. [49] the authors use the chromoelectric and chromomagnetic fields $(i, j=1,2,3)$,

$$
E_{i}=G_{i 0} \quad \text { and } \quad B_{i}=-\frac{1}{2} \epsilon_{i j k} G^{j k},
$$

to write down all the nonrelativistic low-dimension twogluon glueball operators,

$$
\begin{array}{ll}
0^{++} & \vec{E}_{a}^{2} \pm \vec{B}_{a}^{2}, \\
0^{-+} & \vec{E}_{a} \cdot \vec{B}_{a}, \\
1^{-+} & \vec{E}_{a} \times \vec{B}_{a}, \\
2^{++} & \mathcal{S}^{\prime}\left[E_{a}^{i} E_{a}^{j} \pm B_{a}^{i} B_{a}^{j}\right], \\
2^{-+} \mathcal{S}^{\prime} & {\left[E_{a}^{i} B_{a}^{j}-B_{a}^{i} E_{a}^{j}\right],}
\end{array}
$$

where $\mathcal{S}^{\prime}$ denotes symmetrization and subtracting trace terms in the set $\{i j\}$.

We construct their corresponding relativistic currents in order to perform QCD sum rule analyses,

$$
\begin{aligned}
J_{0} & =g_{s}^{2} G_{a}^{\mu \nu} G_{\mu \nu}^{a}, \\
\tilde{J}_{0} & =g_{s}^{2} G_{a}^{\mu \nu} \tilde{G}_{\mu \nu}^{a}, \\
J_{1}^{\alpha \beta} & =g_{s}^{2} G_{a}^{\alpha \mu} \tilde{G}_{\mu}^{a, \beta}-\{\alpha \leftrightarrow \beta\}, \\
J_{2}^{\alpha_{1} \alpha_{2}, \beta_{1} \beta_{2}} & =\mathcal{S}\left[g_{s}^{2} G_{a}^{\alpha_{1} \beta_{1}} G^{a, \alpha_{2} \beta_{2}}\right], \\
\tilde{J}_{2}^{\alpha_{1} \alpha_{2}, \beta_{1} \beta_{2}} & =\mathcal{S}\left[g_{s}^{2} G_{a}^{\alpha_{1} \beta_{1}} \tilde{G}^{a, \alpha_{2} \beta_{2}}\right] .
\end{aligned}
$$


We shall explicitly prove in Appendix that the third current $J_{1}^{\alpha \beta}$ vanishes, suggesting that the "ground-state" two-gluon glueball of $J^{P C}=1^{-+}$does not exist within the relativistic framework.

The former two currents $J_{0}$ of $J^{P C}=0^{++}$and $\tilde{J}_{0}$ of $J^{P C}=0^{-+}$couple to the $J^{P C}=0^{++}$and $0^{-+}$two-gluon glueballs $\left|\mathrm{GG} ; J^{P C}\right\rangle$, respectively,

$$
\begin{aligned}
& \left\langle 0\left|J_{0}\right| \mathrm{GG} ; 0^{++}\right\rangle=f_{0^{++}}, \\
& \left\langle 0\left|\tilde{J}_{0}\right| \mathrm{GG} ; 0^{-+}\right\rangle=f_{0^{-+}},
\end{aligned}
$$

where $f_{0^{++}}$and $f_{0^{-+}}$are decay constants. In addition, the current $J_{0}$ has a partner,

$$
J_{0}^{\prime}=g_{s}^{2} \tilde{G}_{a}^{\mu \nu} \tilde{G}_{\mu \nu}^{a},
$$

whose sum rule result is the same as that of $J_{0}$.

The latter two currents $J_{2}^{\alpha_{1} \alpha_{2}, \beta_{1} \beta_{2}}$ and $\tilde{J}_{2}^{\alpha_{1} \alpha_{2}, \beta_{1} \beta_{2}}$ couple to the $J^{P C}=2^{++}$and $2^{-+}$glueballs through

$$
\begin{aligned}
& \left\langle 0\left|J_{2}^{\cdots}\right| \mathrm{GG} ; 2^{++}\right\rangle=i f_{2^{++}} \mathcal{S}\left[\epsilon^{\alpha_{i} \beta_{i} \mu_{i} \nu_{i}} p_{\nu_{i}}\right]^{2} \epsilon_{\mu_{1} \mu_{2}}, \\
& \left\langle 0\left|\tilde{J}_{2}^{\cdots}\right| \mathrm{GG} ; 2^{-+}\right\rangle=i f_{2^{-+}} \mathcal{S}\left[\epsilon^{\alpha_{i} \beta_{i} \mu_{i} \nu_{i}} p_{\nu_{i}}\right]^{2} \epsilon_{\mu_{1} \mu_{2}} .
\end{aligned}
$$

The current $J_{2}^{\alpha_{1} \alpha_{2}, \beta_{1} \beta_{2}}$ also has a partner,

$$
J_{2}^{\prime \alpha_{1} \alpha_{2}, \beta_{1} \beta_{2}}=\mathcal{S}\left[g_{s}^{2} \tilde{G}_{a}^{\alpha_{1} \beta_{1}} \tilde{G}^{a, \alpha_{2} \beta_{2}}\right] .
$$

whose sum rule result is the same as that of $J_{2}^{\alpha_{1} \alpha_{2}, \beta_{1} \beta_{2}}$.

\section{Three-gluon glueball currents of $\boldsymbol{C}=+$}

In this subsection we use $G_{\mu \nu}^{a}$ and $\tilde{G}_{\mu \nu}^{a}$ to construct three-gluon glueball currents of $C=+$. Some of their corresponding nonrelativistic operators have been constructed in Ref. [49]

$$
\begin{array}{ll}
0^{++} & f^{a b c}\left(\vec{E}_{a} \times \vec{E}_{b}\right) \cdot \vec{B}_{c}, \\
0^{-+} & f^{a b c}\left(\vec{E}_{a} \times \vec{E}_{b}\right) \cdot \vec{E}_{c}, \\
1^{++} & f^{a b c}\left(\vec{B}_{a} \cdot \vec{E}_{b}\right) \vec{E}_{c} \\
1^{-+} & f^{a b c}\left(\vec{B}_{a} \cdot \vec{E}_{b}\right) \vec{B}_{c} \\
2^{++} & f^{a b c} \mathcal{S}^{\prime}\left[\left(\vec{B}_{a} \times \vec{B}_{b}\right)^{i} B_{c}^{j}\right]+\cdots \\
2^{-+} & f^{a b c} \mathcal{S}^{\prime}\left[\left(\vec{E}_{a} \times \vec{E}_{b}\right)^{i} E_{c}^{j}\right]+\cdots
\end{array}
$$

We further construct their corresponding relativistic currents as follows:

$$
\begin{aligned}
& \eta_{0}=f^{a b c} g_{s}^{3} G_{a}^{\mu \nu} G_{b, \nu \rho} G_{c, \mu}^{\rho}, \\
& \tilde{\eta}_{0}=f^{a b c} g_{s}^{3} \tilde{G}_{a}^{\mu \nu} \tilde{G}_{b, \nu \rho} \tilde{G}_{c, \mu}^{\rho},
\end{aligned}
$$

$$
\begin{aligned}
\eta_{1}^{\alpha \beta} & =f^{a b c} g_{s}^{3} \tilde{G}_{a}^{\mu \nu} G_{b, \mu \nu} \tilde{G}_{c}^{\alpha \beta}, \\
\tilde{\eta}_{1}^{\alpha \beta} & =f^{a b c} g_{s}^{3} \tilde{G}_{a}^{\mu \nu} G_{b, \mu \nu} G_{c}^{\alpha \beta}, \\
\eta_{2}^{\alpha_{1} \alpha_{2}, \beta_{1} \beta_{2}} & =f^{a b c} \mathcal{S}\left[g_{s}^{3} G_{a}^{\alpha_{1} \beta_{1}} G_{b}^{\alpha_{2} \mu} G_{c, \mu}^{\beta_{2}}-\left\{\alpha_{2} \leftrightarrow \beta_{2}\right\}\right], \\
\tilde{\eta}_{2}^{\alpha_{1} \alpha_{2}, \beta_{1} \beta_{2}} & =f^{a b c} \mathcal{S}\left[g_{s}^{3} \tilde{G}_{a}^{\alpha_{1} \beta_{1}} \tilde{G}_{b}^{\alpha_{2} \mu} \tilde{G}_{c, \mu}^{\beta_{2}}-\left\{\alpha_{2} \leftrightarrow \beta_{2}\right\}\right] .
\end{aligned}
$$

We shall explicitly prove in Appendix that the third and fourth currents $\eta_{1}^{\alpha \beta}$ and $\tilde{\eta}_{1}^{\alpha \beta}$ both vanish, suggesting that the "ground-state" three-gluon glueballs of $J^{P C}=1^{++}$ and $1^{-+}$do not exist within the relativistic framework.

The former two currents $\eta_{0}$ of $J^{P C}=0^{++}$and $\tilde{\eta}_{0}$ of $J^{P C}=0^{-+}$couple to the $J^{P C}=0^{++}$and $0^{-+}$three-gluon glueballs $\left|\mathrm{GGG} ; J^{P C}\right\rangle$, respectively,

$$
\begin{aligned}
& \left\langle 0\left|\eta_{0}\right| \mathrm{GGG} ; 0^{++}\right\rangle=f_{0^{++}}^{\prime}, \\
& \left\langle 0\left|\tilde{\eta}_{0}\right| \mathrm{GGG} ; 0^{-+}\right\rangle=f_{0^{-+}}^{\prime} .
\end{aligned}
$$

The latter two currents $\eta_{2}^{\alpha_{1} \alpha_{2}, \beta_{1} \beta_{2}}$ and $\tilde{\eta}_{2}^{\alpha_{1} \alpha_{2}, \beta_{1} \beta_{2}}$ couple to the $J^{P C}=2^{++}$and $2^{-+}$glueballs through

$$
\begin{gathered}
\left\langle 0\left|\eta_{2}^{\cdots}\right| \mathrm{GGG} ; 2^{++}\right\rangle=i f_{2^{++}}^{\prime} \mathcal{S}\left[\epsilon^{\alpha_{i} \beta_{i} \mu_{i} \nu_{i}} p_{\nu_{i}}\right]^{2} \epsilon_{\mu_{1} \mu_{2}}, \\
\left\langle 0\left|\tilde{\eta}_{2}^{\cdots}\right| \mathrm{GGG} ; 2^{-+}\right\rangle=i f_{2^{-+}}^{\prime} \mathcal{S}\left[\epsilon^{\alpha_{i} \beta_{i} \mu_{i} \nu_{i}} p_{\nu_{i}}\right]^{2} \epsilon_{\mu_{1} \mu_{2}} .
\end{gathered}
$$

\section{QCD SUM RULE ANALYSES}

In this section we use the two-gluon glueball currents $J_{0}$, $\tilde{J}_{0}, J_{2}^{\alpha_{1} \alpha_{2}, \beta_{1} \beta_{2}}$, and $\tilde{J}_{2}^{\alpha_{1} \alpha_{2}, \beta_{1} \beta_{2}}$ as well as the three-gluon glueball currents $\eta_{0}, \tilde{\eta}_{0}, \eta_{2}^{\alpha_{1} \alpha_{2}, \beta_{1} \beta_{2}}$, and $\tilde{\eta}_{2}^{\alpha_{1} \alpha_{2}, \beta_{1} \beta_{2}}$ to perform QCD sum rule analyses. This method has been widely applied in the field of hadron phenomenology $[52,53]$ to study various exotic hadrons [54-56]; all the above spin-2 currents have four Lorentz indices with certain symmetries, so that they couple to both positive- and negative-parity glueballs simultaneously. We refer to Ref. [50] for detailed discussions.

We take the current $\tilde{J}_{0}$ defined in Eq. (14) as an example, and calculate its two-point correlation function

$$
\Pi\left(q^{2}\right) \equiv i \int d^{4} x e^{i q x}\left\langle 0\left|\mathbf{T}\left[\tilde{J}_{0}(x) \tilde{J}_{0}^{\dagger}(0)\right]\right| 0\right\rangle,
$$

separately at hadron and quark-gluon levels.

At the hadron level we express Eq. (35) using the dispersion relation as

$$
\Pi\left(q^{2}\right)=\int_{0}^{\infty} \frac{\rho(s)}{s-q^{2}-i \varepsilon} d s,
$$


with $\rho(s)=\operatorname{Im} \Pi(s) / \pi$ the spectral density. It is parametrized using one pole dominance for the ground state $X$ as well as the continuum contribution,

$$
\begin{aligned}
\rho(s) & \equiv \sum_{n} \delta\left(s-M_{n}^{2}\right)\left\langle 0\left|\tilde{J}_{0}\right| n\right\rangle\left\langle n\left|\tilde{J}_{0}^{\dagger}\right| 0\right\rangle \\
& =f_{X}^{2} \delta\left(s-M_{X}^{2}\right)+\text { continuum. }
\end{aligned}
$$

At the quark-gluon level we insert Eq. (14) into Eq. (35), and calculate it using the method of operator product expansion (OPE). After performing the Borel transformation to Eq. (36) at both hadron and quark-gluon levels, we approximate the continuum using the spectral density above a threshold value $s_{0}$, and obtain

$$
\Pi\left(s_{0}, M_{B}^{2}\right) \equiv f_{X}^{2} e^{-M_{X}^{2} / M_{B}^{2}}=\int_{0}^{s_{0}} e^{-s / M_{B}^{2}} \rho(s) d s .
$$

This equation can be used to further calculate the mass of $X$ through

$$
M_{X}^{2}\left(s_{0}, M_{B}\right)=\frac{\int_{0}^{s_{0}} e^{-s / M_{B}^{2}} S \rho(s) d s}{\int_{0}^{s_{0}} e^{-s / M_{B}^{2}} \rho(s) d s} .
$$

Since the gluon field strength tensor $G_{\mu \nu}^{a}$ is defined as

$$
G_{\mu \nu}^{a}=\partial_{\mu} A_{\nu}^{a}-\partial_{\nu} A_{\mu}^{a}+g_{s} f^{a b c} A_{b, \mu} A_{c, \nu},
$$

it can be naturally separated into two parts. As shown in Fig. 1, we depict the former two terms using the singlegluon line, and the latter one term using the double-gluon line with a red vertex [see also diagram Fig. 2(c-3)]. Here $A_{\mu}^{a}$ is the gluon field, whose propagator is [57]

$$
\begin{aligned}
\left\langle 0\left|\mathbf{T}\left[A_{\mu}^{a}(x) A_{\nu}^{b}(y)\right]\right| 0\right\rangle= & \frac{\delta^{a b} g_{\mu \nu}}{4 \pi^{2}(x-y)^{2}} \\
& +\frac{g_{s} \ln \left(-(x-y)^{2}\right)}{8 \pi^{2}} f^{a b c} G_{c, \mu \nu}(0) \\
& -\frac{g_{s} g_{\mu \nu} x^{\alpha} y^{\beta}}{8 \pi^{2}(x-y)^{2}} f^{a b c} G_{c, \alpha \beta}(0) .
\end{aligned}
$$

We work in the fixed-point gauge so that

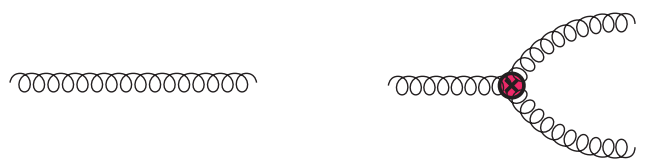

(a)

(b)

FIG. 1. The gluon field strength tensor $G_{\mu \nu}^{a}=\partial_{\mu} A_{\nu}^{a}-$ $\partial_{\nu} A_{\mu}^{a}+g_{s} f^{a b c} A_{b, \mu} A_{c, \nu}$, naturally separated into two parts (a) and (b).

$$
A_{\mu}^{a}(x) \approx-\frac{1}{2} x^{\nu} G_{\mu \nu}^{a}(0)
$$

In the present study we consider the Feynman diagrams depicted in Fig. 2 (for three-gluon glueballs), and calculate OPEs up to dimension eight $(D=8)$ condensates. We take into account the perturbative term, the two-gluon condensate $\left\langle g_{s}^{2} G G\right\rangle$, the three-gluon condensate $\left\langle g_{s}^{3} G^{3}\right\rangle$, and the $D=8$ condensate $\left\langle g_{s}^{2} G G\right\rangle^{2}$,

$$
\begin{aligned}
\Pi_{\left|\mathrm{GG} ; 0^{++}\right\rangle}\left(s_{0}, M_{B}^{2}\right)= & \int_{0}^{s_{0}}\left(32 \alpha_{s}^{2} s^{2}+60 \alpha_{s}^{2}\left\langle g_{s}^{2} G G\right\rangle\right) e^{-s / M_{B}^{2}} d s \\
& +24 \pi \alpha_{s}\left\langle g_{s}^{3} G^{3}\right\rangle, \\
\Pi_{\left|\mathrm{GG} ; 2^{++}\right\rangle}\left(s_{0}, M_{B}^{2}\right)= & \int_{0}^{s_{0}}\left(\frac{2 \alpha_{s}^{2}}{15} s^{2}-\frac{5 \alpha_{s}^{2}\left\langle g_{s}^{2} G G\right\rangle}{24}\right) e^{-s / M_{B}^{2}} d s \\
& +\frac{\pi \alpha_{s}\left\langle g_{s}^{3} G^{3}\right\rangle}{3}, \\
\Pi_{\left|\mathrm{GG} ; 0^{-+}\right\rangle}\left(s_{0}, M_{B}^{2}\right)= & \int_{0}^{s_{0}} 32 \alpha_{s}^{2} s^{2} e^{-s / M_{B}^{2}} d s-40 \pi \alpha_{s}\left\langle g_{s}^{3} G^{3}\right\rangle,
\end{aligned}
$$

$$
\begin{aligned}
\Pi_{\left|\mathrm{GG} ; 2^{-+}\right\rangle}\left(s_{0}, M_{B}^{2}\right)= & \int_{0}^{s_{0}}\left(\frac{2 \alpha_{s}^{2}}{5} s^{2}+\frac{\alpha_{s}^{2}\left\langle g_{s}^{2} G G\right\rangle}{12}\right) e^{-s / M_{B}^{2}} d s \\
& -\frac{\pi \alpha_{s}\left\langle g_{s}^{3} G^{3}\right\rangle}{2}
\end{aligned}
$$

$$
\begin{aligned}
& \Pi_{\left|\mathrm{GGG} ; 0^{++}\right\rangle}\left(s_{0}, M_{B}^{2}\right)= \int_{0}^{s_{0}}\left(\frac{3 \alpha_{s}^{3}}{10 \pi} s^{4}+\frac{135 \alpha_{s}^{3}\left\langle g_{s}^{2} G G\right\rangle}{32 \pi} s^{2}\right. \\
&\left.-\frac{81 \alpha_{s}^{2}\left\langle g_{s}^{3} G^{3}\right\rangle}{2} s\right) e^{-s / M_{B}^{2}} d s \\
& \Pi_{\left|\mathrm{GGG} ; 2^{++}\right\rangle}\left(s_{0}, M_{B}^{2}\right) \int_{0}^{s_{0}}\left(\frac{2 \alpha_{s}^{3}}{315 \pi} s^{4}+\frac{\alpha_{s}^{2}\left\langle g_{s}^{2} G G\right\rangle}{15} s^{2}\right. \\
&\left.\quad+\frac{53 \alpha_{s}^{3}\left\langle g_{s}^{2} G G\right\rangle}{320 \pi} s^{2}+\frac{\alpha_{s}^{2}\left\langle g_{s}^{3} G^{3}\right\rangle}{3} s\right) e^{-s / M_{B}^{2}} d s
\end{aligned}
$$

$$
\begin{aligned}
\Pi_{\left|\mathrm{GGG} ; 0^{-+}\right\rangle}\left(s_{0}, M_{B}^{2}\right)= & \int_{0}^{s_{0}}\left(\frac{3 \alpha_{s}^{3}}{10 \pi} s^{4}+\frac{135 \alpha_{s}^{3}\left\langle g_{s}^{2} G G\right\rangle}{32 \pi} s^{2}\right. \\
& \left.+\frac{27 \alpha_{s}^{2}\left\langle g_{s}^{3} G^{3}\right\rangle}{2} s\right) e^{-s / M_{B}^{2}} d s
\end{aligned}
$$




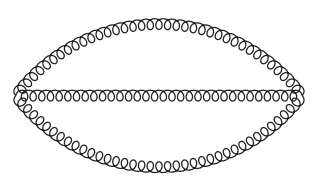

(a)

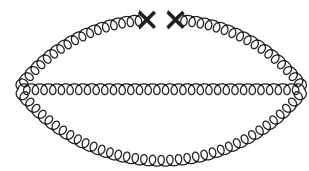

$(b-1)$

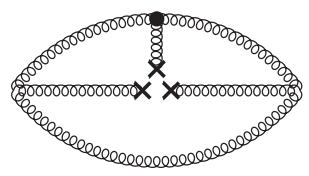

$(c-1)$

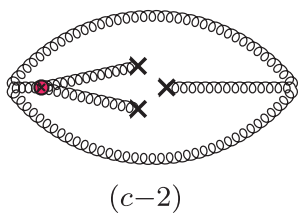

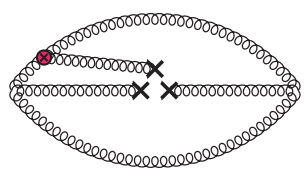

$(c-3)$

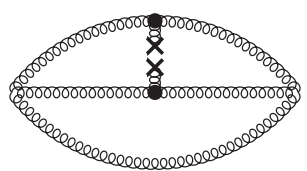

$(d)$
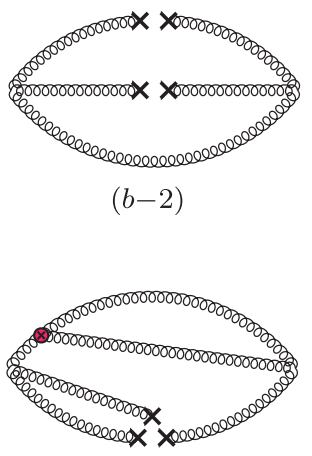

$(c-4)$

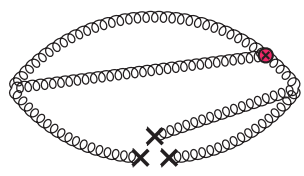

$(c-5)$

FIG. 2. Feynman diagrams for three-gluon glueball currents, including the perturbative term, the two-gluon condensate $\left\langle g_{s}^{2} G G\right\rangle$, the three-gluon condensate $\left\langle g_{s}^{3} G^{3}\right\rangle$, and the $D=8$ condensate $\left\langle g_{s}^{2} G G\right\rangle^{2}$. Diagrams $(a)$ and $(b-i)$ are proportional to $\alpha_{s}^{3} \times g_{s}^{0}$, diagrams $(c-i)$ are proportional to $\alpha_{s}^{3} \times g_{s}^{1}$, and diagram $(d)$ is proportional to $\alpha_{s}^{3} \times g_{s}^{2}$.

$$
\begin{aligned}
& \Pi_{\left|\mathrm{GGG} ; 2^{-+}\right\rangle}\left(s_{0}, M_{B}^{2}\right) \\
& =\int_{0}^{s_{0}}\left(\frac{2 \alpha_{s}^{3}}{315 \pi} s^{4}-\frac{\alpha_{s}^{2}\left\langle g_{s}^{2} G G\right\rangle}{15} s^{2}\right. \\
& \left.\quad+\frac{57 \alpha_{s}^{3}\left\langle g_{s}^{2} G G\right\rangle}{320 \pi} s^{2}+\frac{5 \alpha_{s}^{2}\left\langle g_{s}^{3} G^{3}\right\rangle}{4} s\right) e^{-s / M_{B}^{2}} d s .
\end{aligned}
$$

In the calculations we have considered all the diagrams proportional to $\alpha_{s}^{n} \times g_{s}^{0}$ and $\alpha_{s}^{n} \times g_{s}^{1}(n=2$ for two-gluon glueballs and $n=3$ for three-gluon glueballs); however, there are so many diagrams proportional to $\alpha_{s}^{n} \times g_{s}^{2}$, so we have only taken into account one of them. Specifically, we find that all the $D=8$ terms proportional to $\left\langle g_{s}^{2} G G\right\rangle^{2}$ vanish, so the convergence of the above OPE series are quite good.

In Ref. [22] the authors studied $J^{P C}=0^{++}$three-gluon glueballs using the current $\eta_{0}$ defined in Eq. (25), where they calculated the Feynman diagrams depicted in Figs. 2 $(a, b-i, c-1, c-2)$. In Ref. [24] the authors studied $J^{P C}=0^{-+}$three-gluon glueballs using the current $\tilde{\eta}_{0}$ defined in Eq. (26), where they calculated the diagrams depicted in Figs. $2(a, b-i, c-i)$. Their calculations are done (mainly) by hand. In the present study we use the software Mathematica with the package FeynCalc, and we can obtain exactly the same results for these diagrams. In
Refs. [16,21,23] the authors studied $J^{P C}=0^{++}$and $0^{-+}$ two-gluon glueballs using the currents $J_{0}$ and $\tilde{J}_{0}$ defined in Eqs. (13) and (14), where they calculated more diagrams than those calculated in the present study. However, such calculations are too complicated to be applied to threegluon glueballs, and we still calculate similar diagrams as those depicted in Fig. 2 for two-gluon glueballs to make the present study unified as a whole.

For completeness, we also investigate the following three-gluon glueball currents of $C=-$,

$$
\begin{aligned}
\xi_{1}^{\alpha \beta} & =d^{a b c} g_{s}^{3} G_{a}^{\mu \nu} G_{b, \mu \nu} G_{c}^{\alpha \beta}, \\
\tilde{\xi}_{1}^{\alpha \beta} & =d^{a b c} g_{s}^{3} G_{a}^{\mu \nu} G_{b, \mu \nu} \tilde{G}_{c}^{\alpha \beta}, \\
\xi_{2}^{\alpha_{1} \alpha_{2}, \beta_{1} \beta_{2}} & =d^{a b c} \mathcal{S}\left[g_{s}^{3} \tilde{G}_{a}^{\alpha_{1} \beta_{1}} G_{b}^{\alpha_{2} \mu} \tilde{G}_{c, \mu}^{\beta_{2}}-\left\{\alpha_{2} \leftrightarrow \beta_{2}\right\}\right], \\
\tilde{\xi}_{2}^{\alpha_{1} \alpha_{2}, \beta_{1} \beta_{2}} & =d^{a b c} \mathcal{S}\left[g_{s}^{3} G_{a}^{\alpha_{1} \beta_{1}} \tilde{G}_{b}^{\alpha_{2} \mu} G_{c, \mu}^{\beta_{2}}-\left\{\alpha_{2} \leftrightarrow \beta_{2}\right\}\right], \\
\xi_{3}^{\cdots} & =d^{a b c} \mathcal{S}\left[g_{s}^{3} G_{a}^{\alpha_{1} \beta_{1}} G_{b}^{\alpha_{2} \beta_{2}} G_{c}^{\alpha_{3} \beta_{3}}\right], \\
\tilde{\xi}_{3} \cdots & =d^{a b c} \mathcal{S}\left[g_{s}^{3} \tilde{G}_{a}^{\alpha_{1} \beta_{1}} \tilde{G}_{b}^{\alpha_{2} \beta_{2}} \tilde{G}_{c}^{\alpha_{3} \beta_{3}}\right],
\end{aligned}
$$


where $d^{a b c}$ is the totally symmetric $S U(3)_{C}$ structure constant. Their sum rule equations are

$$
\begin{aligned}
& \Pi_{\left|\mathrm{GGG} ; 1^{+-}\right\rangle}\left(s_{0}, M_{B}^{2}\right) \\
& =\int_{0}^{s_{0}}\left(\frac{4 \alpha_{s}^{3}}{81 \pi} s^{4}+\frac{10 \alpha_{s}^{2}\left\langle g_{s}^{2} G G\right\rangle}{9} s^{2}\right. \\
& \left.+\frac{35 \alpha_{s}^{3}\left\langle g_{s}^{2} G G\right\rangle}{36 \pi} s^{2}+\frac{5 \alpha_{s}^{2}\left\langle g_{s}^{3} G^{3}\right\rangle}{27} s\right) e^{-s / M_{B}^{2}} d s, \\
& \Pi_{\left|\mathrm{GGG} ; 2^{+-}\right\rangle}\left(s_{0}, M_{B}^{2}\right) \\
& =\int_{0}^{s_{0}}\left(\frac{\alpha_{s}^{3}}{324 \pi} s^{4}-\frac{5 \alpha_{s}^{2}\left\langle g_{s}^{2} G G\right\rangle}{108} s^{2}\right. \\
& \left.+\frac{15 \alpha_{s}^{3}\left\langle g_{s}^{2} G G\right\rangle}{128 \pi} s^{2}+\frac{65 \alpha_{s}^{2}\left\langle g_{s}^{3} G^{3}\right\rangle}{216} s\right) e^{-s / M_{B}^{2}} d s, \\
& \Pi_{\left|\mathrm{GGG} ; 3^{+-}\right\rangle}\left(s_{0}, M_{B}^{2}\right) \\
& =\int_{0}^{s_{0}}\left(\frac{5 \alpha_{s}^{3}}{2016 \pi} s^{4}+\frac{\alpha_{s}^{2}\left\langle g_{s}^{2} G G\right\rangle}{16} s^{2}\right. \\
& \left.-\frac{59 \alpha_{s}^{3}\left\langle g_{s}^{2} G G\right\rangle}{512 \pi} s^{2}-\frac{\alpha_{s}^{2}\left\langle g_{s}^{3} G^{3}\right\rangle}{2} s\right) e^{-s / M_{B}^{2}} d s, \\
& \Pi_{\left|\mathrm{GGG} ; 1^{--}\right\rangle}\left(s_{0}, M_{B}^{2}\right) \\
& =\int_{0}^{s_{0}}\left(\frac{4 \alpha_{s}^{3}}{81 \pi} s^{4}-\frac{10 \alpha_{s}^{2}\left\langle g_{s}^{2} G G\right\rangle}{9} s^{2}\right. \\
& \left.+\frac{25 \alpha_{s}^{3}\left\langle g_{s}^{2} G G\right\rangle}{36 \pi} s^{2}+\frac{35 \alpha_{s}^{2}\left\langle g_{s}^{3} G^{3}\right\rangle}{27} s\right) e^{-s / M_{B}^{2}} d s, \\
& \Pi_{\left|\mathrm{GGG} ; 2^{--}\right\rangle}\left(s_{0}, M_{B}^{2}\right) \\
& =\int_{0}^{s_{0}}\left(\frac{\alpha_{s}^{3}}{324 \pi} s^{4}+\frac{5 \alpha_{s}^{2}\left\langle g_{s}^{2} G G\right\rangle}{108} s^{2}\right. \\
& \left.+\frac{15 \alpha_{s}^{3}\left\langle g_{s}^{2} G G\right\rangle}{128 \pi} s^{2}+\frac{5 \alpha_{s}^{2}\left\langle g_{s}^{3} G^{3}\right\rangle}{24} s\right) e^{-s / M_{B}^{2}} d s, \\
& \Pi_{\left|\mathrm{GGG} ; 3^{--}\right\rangle}\left(s_{0}, M_{B}^{2}\right) \\
& =\int_{0}^{s_{0}}\left(\frac{5 \alpha_{s}^{3}}{2016 \pi} s^{4}-\frac{\alpha_{s}^{2}\left\langle g_{s}^{2} G G\right\rangle}{16} s^{2}\right. \\
& \left.-\frac{49 \alpha_{s}^{3}\left\langle g_{s}^{2} G G\right\rangle}{1536 \pi} s^{2}-\frac{11 \alpha_{s}^{2}\left\langle g_{s}^{3} G^{3}\right\rangle}{432} s\right) e^{-s / M_{B}^{2}} d s .
\end{aligned}
$$

The above three-gluon glueball currents of $C=-$ have been systematically studied in Ref. [50], but there we did not calculate the Feynman diagrams depicted in Figs. 2 $(c-3, c-4, c-5)$. Similar to Eqs. (43)-(50), we find all the $D=8$ terms proportional to $\left\langle g_{s}^{2} G G\right\rangle^{2}$ vanish, so the convergence of the above OPE series are also quite good.

We shall use the above sum rule equations to perform numerical analyses in the next section.

\section{NUMERICAL ANALYSES}

In this section we perform numerical analyses using the sum rules given in Eqs. (43)-(50) and (57)-(62). The glueball mass $M_{X}$ depends significantly on the gluon condensates $\left\langle g_{s}^{2} G G\right\rangle$ and $\left\langle g_{s}^{3} G^{3}\right\rangle$, both of which are still not precisely known. In the present study we use the following values for these parameters $[58,59]$

$$
\begin{aligned}
\left\langle\alpha_{s} G G\right\rangle & =(6.35 \pm 0.35) \times 10^{-2} \mathrm{GeV}^{4}, \\
\left\langle g_{s}^{3} G^{3}\right\rangle & =\left\langle\alpha_{s} G G\right\rangle \times(8.2 \pm 1.0) \mathrm{GeV}^{2} .
\end{aligned}
$$

Additionally, we use the following value for the strong coupling constant at the QCD scale $\Lambda_{\mathrm{QCD}}=300 \mathrm{MeV}$ [60],

$$
\alpha_{s}\left(Q^{2}\right)=\frac{4 \pi}{11 \ln \left(Q^{2} / \Lambda_{\mathrm{QCD}}^{2}\right)} .
$$

We still take the current $\tilde{J}_{0}$ as an example, and use Eq. (39) to calculate the mass of $\left|\mathrm{GG} ; 0^{-+}\right\rangle$. It depends on two free parameters, the Borel mass $M_{B}$ and the threshold value $s_{0}$. We use two criteria to determine the Borel window. The first criterion is to ensure the convergence of OPE by requiring: a) the $\alpha_{s}^{2} \times g_{s}^{2}$ term $\alpha_{s}^{2}\left\langle g_{s}^{2} G G\right\rangle$ to be less than $5 \%$, and b) the $D=6$ term $\alpha_{s}\left\langle g_{s}^{3} G^{3}\right\rangle$ to be less than $10 \%$

$$
\begin{gathered}
\mathrm{CVG}_{A} \equiv\left|\frac{\Pi^{9_{s}^{n=6}}\left(s_{0}, M_{B}^{2}\right)}{\Pi\left(s_{0}, M_{B}^{2}\right)}\right| \leq 5 \%, \\
\mathrm{CVG}_{B} \equiv\left|\frac{\Pi^{\mathrm{D}=6}\left(s_{0}, M_{B}^{2}\right)}{\Pi\left(s_{0}, M_{B}^{2}\right)}\right| \leq 10 \% .
\end{gathered}
$$

As shown in Fig. 3 using the dashed curves, we determine the lower limit of $M_{B}$ to be $M_{B}^{2} \geq 3.28 \mathrm{GeV}^{2}$ when setting $s_{0}=9.0 \mathrm{GeV}^{2}$.

The above condition is the cornerstone of a reliable sum rule analysis, where we have taken into account two terms

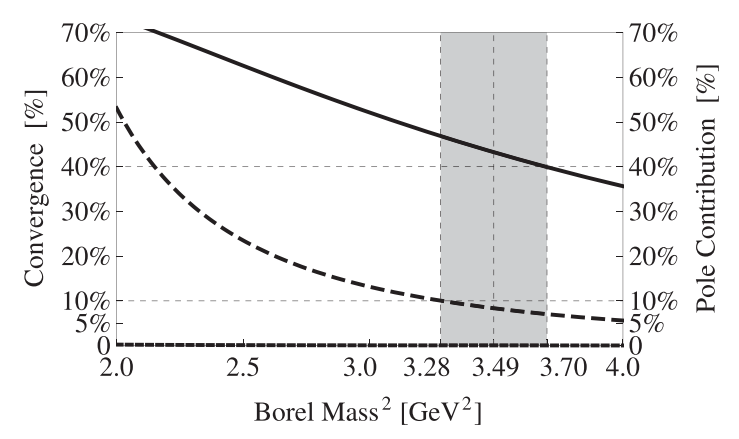

FIG. 3. $\mathrm{CVG}_{A}$ [short-dashed curve, defined in Eq. (65)], $\mathrm{CVG}_{B}$ [long-dashed curve, defined in Eq. (66)], and PC [solid curve, defined in Eq. (69)] as functions of the Borel mass $M_{B}$. The current $\tilde{J}_{0}$ is used here when setting $s_{0}=9.0 \mathrm{GeV}^{2}$. 

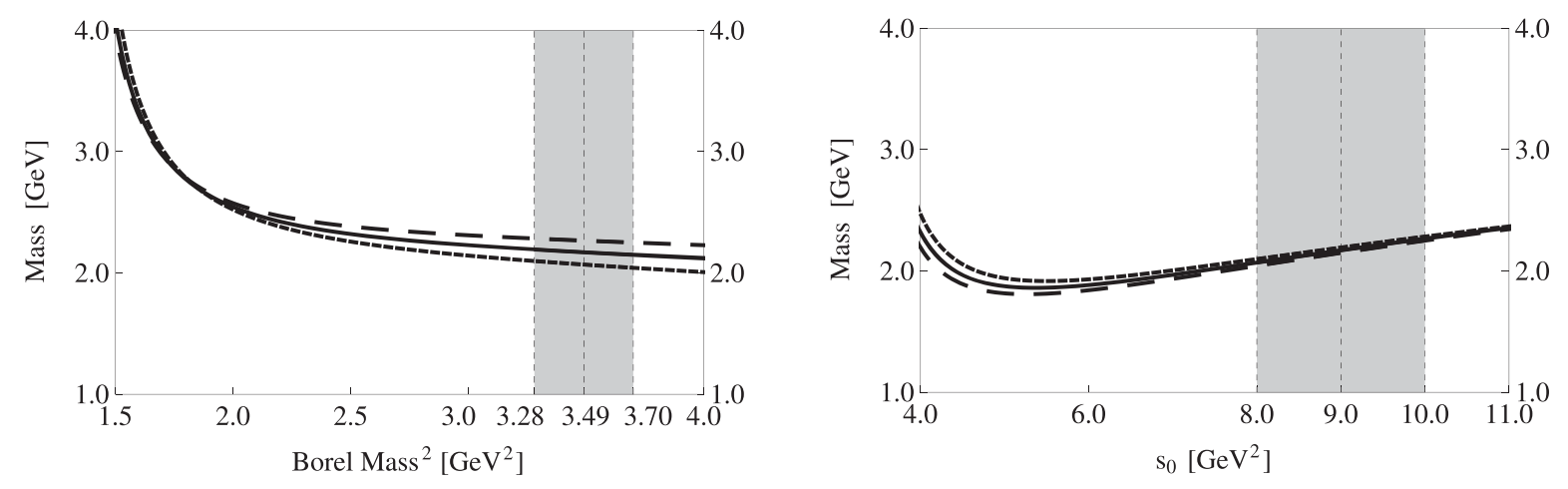

FIG. 4. Mass of the two-gluon glueball $\left|\mathrm{GG} ; 0^{-+}\right\rangle$as a function of the Borel mass $M_{B}$ (left) and the threshold value $s_{0}$ (right), calculated using the current $\tilde{J}_{0}$. In the left panel the short-dashed/solid/long-dashed curves are obtained by setting $s_{0}=8.0 / 9.0 / 10.0 \mathrm{GeV}^{2}$, respectively. In the right panel the short-dashed/solid/long-dashed curves are obtained by setting $M_{B}^{2}=3.28 / 3.49 / 3.70 \mathrm{GeV}^{2}$, respectively.

because the OPE is expanded in two directions; the dimension of condensates and the coupling constant $g_{s}$. Equations (65) and (66) are for two-gluon glueball currents, and the conditions for three-gluon glueball currents are

$$
\begin{gathered}
\mathrm{CVG}_{A}^{\prime} \equiv\left|\frac{\Pi_{s}^{g_{s}^{n=8}}\left(s_{0}, M_{B}^{2}\right)}{\Pi\left(s_{0}, M_{B}^{2}\right)}\right| \leq 5 \%, \\
\mathrm{CVG}_{B}^{\prime} \equiv\left|\frac{\Pi^{\mathrm{D}=6}\left(s_{0}, M_{B}^{2}\right)}{\Pi\left(s_{0}, M_{B}^{2}\right)}\right| \leq 10 \% .
\end{gathered}
$$

The second criterion is to insure the one-pole-dominance assumption by requiring the pole contribution (PC) to be larger than $40 \%$,

$$
\mathrm{PC} \equiv\left|\frac{\Pi\left(s_{0}, M_{B}^{2}\right)}{\Pi\left(\infty, M_{B}^{2}\right)}\right| \geq 40 \% .
$$

As shown in Fig. 3 (using the solid curve), we determine the upper limit of $M_{B}$ to be $M_{B}^{2} \leq 3.70 \mathrm{GeV}^{2}$ when setting $s_{0}=9.0 \mathrm{GeV}^{2}$.

Altogether we determine the Borel window to be $3.28 \mathrm{GeV}^{2} \leq M_{B}^{2} \leq 3.70 \mathrm{GeV}^{2}$ for the fixed threshold value $s_{0}=9.0 \mathrm{GeV}^{2}$. Then we redo the same procedures by changing $s_{0}$, and find that there exists nonvanishing Borel windows as long as $s_{0} \geq s_{0}^{\min }=8.2 \mathrm{GeV}^{2}$. Accordingly, we choose $s_{0}$ to be slightly larger, and determine our working regions to be $8.0 \mathrm{GeV}^{2} \leq s_{0} \leq$ $10.0 \mathrm{GeV}^{2}$ and $3.28 \mathrm{GeV}^{2} \leq M_{B}^{2} \leq 3.70 \mathrm{GeV}^{2}$, where we calculate the mass of $\left|\mathrm{GG} ; 0^{-+}\right\rangle$to be

$$
M_{\left|\mathrm{GG} ; 0^{-+}\right\rangle}=2.17 \pm 0.11 \mathrm{GeV} .
$$

Its central value corresponds to $M_{B}^{2}=3.49 \mathrm{GeV}^{2}$ and $s_{0}=9.0 \mathrm{GeV}^{2}$, its uncertainty comes from the threshold value $s_{0}$, the Borel mass is $M_{B}$, and the gluon condensates are listed in Eqs. (63).
We show $M_{\left|\mathrm{GG} ; 0^{-+}\right\rangle}$in the left panel of Fig. 4 as a function of the Borel mass $M_{B}$, and find it quite stable inside the Borel window $3.28 \mathrm{GeV}^{2} \leq M_{B}^{2} \leq 3.70 \mathrm{GeV}^{2}$. We also show it in the right panel of Fig. 4 as a function of the threshold value $s_{0}$. We find there exists a mass minimum around $s_{0} \sim 5 \mathrm{GeV}^{2}$, and the $s_{0}$ dependence is weak and acceptable inside the working region $8.0 \mathrm{GeV}^{2} \leq s_{0} \leq 10.0 \mathrm{GeV}^{2}$.

Similarly, we use the sum rules given in Eqs. (43)-(50) and (57)-(62) to perform numerical analyses, and calculate masses of two- and three-gluon glueballs systematically. The obtained results are summarized in Table I, where we choose threshold values $s_{0}$ for two-gluon glueballs to be around $9-10 \mathrm{GeV}^{2}$, and those for three-gluon glueballs to be around $33-38 \mathrm{GeV}^{2}$.

\section{SUMMARY AND DISCUSSIONS}

In this paper we study two- and three-gluon glueballs of $C=+$ using the method of QCD sum rules, including

(a) the two-gluon glueballs with the quantum numbers $J^{P C}=0^{ \pm+}, 1^{-+}$, and $2^{ \pm+}$;

(b) the three-gluon glueballs with the quantum numbers $J^{P C}=0^{ \pm+}, 1^{ \pm+}$, and $2^{ \pm+}$.

We systematically construct their interpolating currents, and find that all the spin- 1 currents of $C=+$ vanish, suggesting that the "ground-state" spin-1 glueballs of $C=+$ do not exist within the relativistic framework. This behavior is consistent with lattice QCD calculations [11-14].

We use spin-0 and spin-2 glueball currents to perform QCD sum rule analyses, and calculate the masses of their corresponding spin-0 and spin-2 glueballs. All these spin-2 currents have four Lorentz indices with certain symmetries, so that they couple to both positive- and negative-parity glueballs, which need to be further separated at the hadron level. We refer to Ref. [50] for detailed discussions.

We summarize the obtained results in Table II, which are compared with the lattice QCD results obtained using 
TABLE I. QCD sum rule results of two- and three-gluon glueballs.

\begin{tabular}{lcccccc}
\hline \hline & & & \multicolumn{2}{c}{ Working Regions } & & \\
Glueball & Current & $s_{0}^{\min }\left[\mathrm{GeV}^{2}\right]$ & $s_{0}\left[\mathrm{GeV}^{2}\right]$ & $M_{B}^{2}\left[\mathrm{GeV}^{2}\right]$ & Pole [\%] & Mass [GeV] \\
\hline$\left|\mathrm{GG} ; 0^{++}\right\rangle$ & $J_{0}$ & 7.8 & $9.0 \pm 1.0$ & $3.70-4.19$ & $40-48$ & $1.78_{-0.17}^{+0.14}$ \\
$\left|\mathrm{GG} ; 2^{++}\right\rangle$ & $J_{2}^{\alpha_{1} \alpha_{2}, \beta_{1} \beta_{2}}$ & 8.5 & $10.0 \pm 1.0$ & $3.99-4.60$ & $40-50$ & $1.86_{-0.17}^{+0.14}$ \\
$\left|\mathrm{GG} ; 0^{-+}\right\rangle$ & $\tilde{J}_{0}$ & 8.2 & $9.0 \pm 1.0$ & $3.28-3.70$ & $40-47$ & $2.17_{-0.11}^{+0.11}$ \\
$\left|\mathrm{GG} ; 2^{-+}\right\rangle$ & $\tilde{J}_{2}^{\alpha_{1} \alpha_{2}, \beta_{1} \beta_{2}}$ & 8.1 & $10.0 \pm 1.0$ & $3.27-4.20$ & $40-55$ & $2.24_{-0.11}^{+0.11}$ \\
$\left|\mathrm{GGG} ; 0^{++}\right\rangle$ & $\eta_{0}$ & 31.6 & $33.0 \pm 3.0$ & $7.25-7.61$ & $40-44$ & $4.46_{-0.19}^{+0.17}$ \\
$\left|\mathrm{GGG} ; 2^{++}\right\rangle$ & $\eta_{2}^{\alpha_{1} \alpha_{2}, \beta_{1} \beta_{2}}$ & 16.0 & $35.0 \pm 3.0$ & $4.77-9.04$ & $40-90$ & $4.18_{-0.42}^{+0.19}$ \\
$\left|\mathrm{GGG} ; 0^{-+}\right\rangle$ & $\tilde{\eta}_{0}$ & 17.0 & $33.0 \pm 3.0$ & $4.48-8.13$ & $40-88$ & $4.13_{-0.36}^{+0.18}$ \\
$\left|\mathrm{GGG} ; 2^{-+}\right\rangle$ & $\tilde{\eta}_{2}^{\alpha_{1} \alpha_{2}, \beta_{1} \beta_{2}}$ & 33.1 & $35.0 \pm 3.0$ & $8.10-8.53$ & $40-44$ & $4.29_{-0.22}^{+0.20}$ \\
$\left|\mathrm{GGG} ; 1^{+-}\right\rangle$ & $\xi_{1}^{\alpha \beta}$ & 9.0 & $34.0 \pm 4.0$ & $3.16-9.09$ & $40-99$ & $4.01_{-0.95}^{+0.26}$ \\
$\left|\mathrm{GGG} ; 2^{+-}\right\rangle$ & $\xi_{2}^{\alpha_{1} \alpha_{2}, \beta_{1} \beta_{2}}$ & 32.7 & $35.0 \pm 4.0$ & $7.53-8.09$ & $40-46$ & $4.42_{-0.29}^{+0.24}$ \\
$\left|\mathrm{GGG} ; 3^{+-}\right\rangle$ & $\xi_{3}^{\alpha_{1} \alpha_{2} \alpha_{3}, \beta_{1} \beta_{2} \beta_{3}}$ & 30.2 & $33.0 \pm 4.0$ & $7.69-8.40$ & $40-47$ & $4.30_{-0.26}^{+0.23}$ \\
$\left|\mathrm{GGG} ; 1^{--}\right\rangle$ & $\tilde{\xi}_{1}^{\alpha \beta}$ & 31.2 & $34.0 \pm 4.0$ & $5.81-6.77$ & $40-51$ & $4.91_{-0.18}^{+0.20}$ \\
$\left|\mathrm{GGG} ; 2^{--}\right\rangle$ & $\tilde{\xi}_{2}^{\alpha_{1} \alpha_{2}, \beta_{1} \beta_{2}}$ & 19.7 & $36.0 \pm 4.0$ & $5.80-9.47$ & $40-81$ & $4.25_{-0.33}^{+0.22}$ \\
$\left|\mathrm{GGG} ; 3^{--}\right\rangle$ & $\tilde{\xi}_{3}^{\alpha_{1} \alpha_{2} \alpha_{3}, \beta_{1} \beta_{2} \beta_{3}}$ & 35.8 & $38.0 \pm 4.0$ & $6.15-7.22$ & $40-49$ & $5.59_{-0.22}^{+0.33}$ \\
\hline \hline
\end{tabular}

TABLE II. Masses of two- and three-gluon glueballs, in units of GeV. Our QCD sum rule results are listed in the second column. Lattice QCD results are listed in the third-sixth columns, taken from Refs. [11-13] (quenched) and Ref. [14] (unquenched).

\begin{tabular}{lccccc}
\hline \hline Glueball & QCD sum rules & Ref. [11] & Ref. [12] & Ref. [13] & Ref. [14] \\
\hline$\left|\mathrm{GG} ; 0^{++}\right\rangle$ & $1.78_{-0.17}^{+0.14}$ & $1.71 \pm 0.05 \pm 0.08$ & $1.73 \pm 0.05 \pm 0.08$ & $1.48 \pm 0.03 \pm 0.07$ & $1.80 \pm 0.06$ \\
$\left|\mathrm{GG} ; 2^{++}\right\rangle$ & $1.86_{-0.17}^{+0.14}$ & $2.39 \pm 0.03 \pm 0.12$ & $2.40 \pm 0.03 \pm 0.12$ & $2.15 \pm 0.03 \pm 0.10$ & $2.62 \pm 0.05$ \\
$\left|\mathrm{GG} ; 0^{-+}\right\rangle$ & $2.17_{-0.11}^{+0.11}$ & $2.56 \pm 0.04 \pm 0.12$ & $2.59 \pm 0.04 \pm 0.13$ & $2.25 \pm 0.06 \pm 0.10$ & $\ldots$ \\
$\left|\mathrm{GG} ; 2^{-+}\right\rangle$ & $2.24_{-0.11}^{+0.11}$ & $3.04 \pm 0.04 \pm 0.15$ & $3.10 \pm 0.03 \pm 0.15$ & $2.78 \pm 0.05 \pm 0.13$ & $3.46 \pm 0.32$ \\
$\left|\mathrm{GGG} ; 0^{++}\right\rangle$ & $4.46_{-0.19}^{+0.17}$ & $\ldots$ & $2.67 \pm 0.18 \pm 0.13$ & $2.76 \pm 0.03 \pm 0.12$ & $3.76 \pm 0.24$ \\
$\left|\mathrm{GGG} ; 2^{++}\right\rangle$ & $4.18_{-0.42}^{+0.19}$ & $\ldots$ & $\ldots$ & $2.88 \pm 0.10 \pm 0.13$ & $\ldots$ \\
$\left|\mathrm{GGG} ; 0^{-+}\right\rangle$ & $4.13_{-0.36}^{+0.18}$ & $\ldots$ & $3.64 \pm 0.06 \pm 0.18$ & $3.37 \pm 0.15 \pm 0.15$ & $4.49 \pm 0.59$ \\
$\left|\mathrm{GGG} ; 2^{-+}\right\rangle$ & $4.29_{-0.22}^{+0.20}$ & $\ldots$ & $\cdots$ & $3.48 \pm 0.14 \pm 0.16$ & $\cdots$ \\
$\left|\mathrm{GGG} ; 1^{+-}\right\rangle$ & $4.01_{-0.95}^{+0.26}$ & $2.98 \pm 0.03 \pm 0.14$ & $2.94 \pm 0.03 \pm 0.14$ & $2.67 \pm 0.07 \pm 0.12$ & $3.27 \pm 0.34$ \\
$\left|\mathrm{GGG} ; 2^{+-}\right\rangle$ & $4.42_{-0.29}^{+0.24}$ & $4.23 \pm 0.05 \pm 0.20$ & $4.14 \pm 0.05 \pm 0.20$ & $\ldots$ & $\ldots$ \\
$\left|\mathrm{GGG} ; 3^{+-}\right\rangle$ & $4.30_{-0.26}^{+0.23}$ & $3.60 \pm 0.04 \pm 0.17$ & $3.55 \pm 0.04 \pm 0.17$ & $3.27 \pm 0.09 \pm 0.15$ & $3.85 \pm 0.35$ \\
$\left|\mathrm{GGG} ; 1^{--}\right\rangle$ & $4.91_{-0.18}^{+0.20}$ & $3.83 \pm 0.04 \pm 0.19$ & $3.85 \pm 0.05 \pm 0.19$ & $3.24 \pm 0.33 \pm 0.15$ & $\ldots$ \\
$\left|\mathrm{GGG} ; 2^{--}\right\rangle$ & $4.25_{-0.33}^{+0.22}$ & $4.01 \pm 0.05 \pm 0.20$ & $3.93 \pm 0.04 \pm 0.19$ & $3.66 \pm 0.13 \pm 0.17$ & $4.59 \pm 0.74$ \\
$\left|\mathrm{GGG} ; 3^{--}\right\rangle$ & $5.59_{-0.22}^{+0.33}$ & $4.20 \pm 0.05 \pm 0.20$ & $4.13 \pm 0.09 \pm 0.20$ & $4.33 \pm 0.26 \pm 0.20$ & $\ldots-$ \\
\hline \hline
\end{tabular}

nonrelativistic-glueball operators [11-14]. For completeness, we also reanalyze the results of $C=-$ three-gluon glueballs (also called odderons), which have been previously studied in Ref. [50]. Thus, a rather complete QCD sum rule study has been done on the lowest-lying glueballs composed of two or three valence gluons. We find that our QCD sum rule results are generally consistent with unquenched lattice QCD results [14].
To end this paper, we briefly discuss possible decay patterns of two- and three-gluon glueballs. The two-gluon glueballs can decay after exciting two quark-antiquark pairs, and recombine into two mesons; however, it is rather difficult to differentiate them from standard $q \bar{q}$ states. The three-gluon glueballs can decay after exciting three quarkantiquark pairs, and recombine into three mesons. Their possible decay patterns are 


$$
\begin{array}{ccc}
0^{-+} \rightarrow & V V P, V V V & (S \text {-wave }), \\
0^{++} \rightarrow & V P P, V V P, V V V & (P \text {-wave }), \\
1^{--} \rightarrow & V P P, V V P, V V V & (S \text {-wave }), \\
1^{+-} \rightarrow P P P, V P P, V V P, V V V & (P \text {-wave }), \\
2^{- \pm} \rightarrow & V V P, V V V & (S \text {-wave }), \\
2^{+ \pm} \rightarrow & V P P, V V P, V V V & (P \text {-wave }), \\
3^{--} \rightarrow & V V V & (S \text {-wave }), \\
3^{+-} \rightarrow & V V P, V V V & (P \text {-wave }),
\end{array}
$$

where $P$ and $V$ denote light vector and pseudoscalar mesons, respectively. Considering their limited decay patterns, the $J^{P C}=0^{-+} / 2^{- \pm} / 3^{ \pm-}$three-gluon glueballs may have relatively smaller widths, and we propose to search for them in their $V V V$ and $V V P$ decay channels in future BESIII, GlueX, LHC, and PANDA experiments.

\section{ACKNOWLEDGMENTS}

We thank Zi-Yang Lin very much for helping us prove that all the spin-1 two- and three-gluon glueball currents of $C=+$ vanish. We thank the anonymous PRD referee very much for checking our formulae and correcting one critical mistake. This project is supported by the National Natural Science Foundation of China under Grants No. 11722540, No. 11975033, No. 12075019, and No. 12070131001, the National Key R\&D Program of China under Contract No. 2020YFA0406400, the Jiangsu Provincial DoubleInnovation Program under Grant No. JSSCRC2021488, and the Fundamental Research Funds for the Central Universities.

\section{APPENDIX: SPIN-1 CURRENTS OF $C=+$}

In this Appendix we prove that the three spin- 1 currents $J_{1}^{\alpha \beta}, \eta_{1}^{\alpha \beta}$, and $\tilde{\eta}_{1}^{\alpha \beta}$ all vanish. Their definitions are given in Eqs. (15), (27), and (28), respectively. For simplicity, we shall not differentiate the superscript and subscript in the following calculations.

Firstly, we investigate the current $J_{1}^{\alpha \beta}$. Due to the Lorentz invariant, we simply assume $\alpha=0$ and $\beta=1 \ldots 3$. Additionally, we need the Lorentz indices $\mu=0 / i$, $\rho=0 / k$, and $\sigma=0 / l$, with $i / k / l=1 \ldots 3$. We obtain

$$
\begin{aligned}
2 J_{1}^{0 \beta} & =2 G_{a}^{0 \mu} \tilde{G}_{a}^{\beta \mu}-\{0 \leftrightarrow \beta\}, \\
& =G_{a}^{0 \mu} G_{a}^{\rho \sigma} \epsilon^{\beta \mu \rho \sigma}-G_{a}^{\beta \mu} G_{a}^{\rho \sigma} \epsilon^{0 \mu \rho \sigma} \\
& =G_{a}^{0 i} G_{a}^{k 0} \epsilon^{\beta i k 0}+G_{a}^{0 i} G_{a}^{0 l} \epsilon^{\beta i 0 l}-G_{a}^{\beta i} G_{a}^{k l} \epsilon^{0 i k l} .
\end{aligned}
$$

After interchanging $i \leftrightarrow k$, the first term turns out to be zero,

$$
G_{a}^{0 i} G_{a}^{k 0} \epsilon^{\beta i k 0}=G_{a}^{0 k} G_{a}^{i 0} \epsilon^{\beta k i 0}=G_{a}^{0 i} G_{a}^{k 0} \epsilon^{\beta k i 0} \rightarrow 0,
$$

as does the second term. The third term is nonzero when $\beta=k$ or $\beta=l$; however, for the case $\beta=k$, we can interchange $i \leftrightarrow l$ and obtain (not sum over $\beta$ here),

$$
G_{a}^{\beta i} G_{a}^{\beta l} \epsilon^{0 i \beta l}=G_{a}^{\beta l} G_{a}^{\beta i} \epsilon^{0 l \beta i}=G_{a}^{\beta i} G_{a}^{\beta l} \epsilon^{0 l \beta i} \rightarrow 0,
$$

similar to the case $\beta=l$. Therefore, the third term is also zero, and the current $J_{1}^{\alpha \beta}$ vanishes.

Secondly, we investigate the current $\eta_{1}^{\alpha \beta}$,

$$
\begin{aligned}
2 \eta_{1}^{\alpha \beta} & =2 f_{a b c} \tilde{G}_{a}^{\mu \nu} G_{b}^{\mu \nu} G_{c}^{\alpha \beta} \\
& =f_{a b c} \epsilon^{\mu \nu \rho \sigma} G_{a}^{\rho \sigma} G_{b}^{\mu \nu} G_{c}^{\alpha \beta} \\
& =f_{a b c} \epsilon^{\mu \nu \rho \sigma} G_{a}^{\mu \nu} G_{b}^{\rho \sigma} G_{c}^{\alpha \beta} \\
& =-f_{a b c} \epsilon^{\mu \nu \rho \sigma} G_{b}^{\mu \nu} G_{a}^{\rho \sigma} G_{c}^{\alpha \beta} \\
& \rightarrow 0 .
\end{aligned}
$$

In the above expressions, we have interchanged $\mu \nu \leftrightarrow \rho \sigma$ and $a \leftrightarrow b$. Similarly, we can prove the current $\tilde{\eta}_{1}^{\alpha \beta}$ to be zero.

One can construct more spin-1 three-gluon glueball currents of $C=+$, such as

$$
\begin{aligned}
& \eta_{1}^{\prime \alpha \beta}=f_{a b c} G_{a}^{\alpha \mu} G_{b}^{\mu \nu} G_{c}^{\nu \beta}-\{\alpha \leftrightarrow \beta\}, \\
& \tilde{\eta}_{1}^{\prime \alpha \beta}=f_{a b c} G_{a}^{\alpha \mu} G_{b}^{\mu \nu} \tilde{G}_{c}^{\nu \beta}-\{\alpha \leftrightarrow \beta\} .
\end{aligned}
$$

It is straightforward to prove the former current $\eta_{1}^{\prime \alpha \beta}$ to be zero,

$$
\begin{aligned}
\eta_{1}^{\prime \alpha \beta} & =f_{a b c} G_{a}^{\alpha \mu} G_{b}^{\mu \nu} G_{c}^{\nu \beta}-\{\alpha \leftrightarrow \beta\} \\
& =f_{a b c} G_{a}^{\alpha \nu} G_{b}^{\nu \mu} G_{c}^{\mu \beta}-\{\alpha \leftrightarrow \beta\} \\
& =-f_{a b c} G_{a}^{\beta \nu} G_{b}^{\nu \mu} G_{c}^{\mu \alpha}+\{\alpha \leftrightarrow \beta\} \\
& =f_{a b c} G_{c}^{\beta \nu} G_{b}^{\nu \mu} G_{a}^{\mu \alpha}-\{\alpha \leftrightarrow \beta\} \\
& =-f_{a b c} G_{c}^{\nu \beta} G_{b}^{\mu \nu} G_{a}^{\alpha \mu}-\{\alpha \leftrightarrow \beta\} \\
& \rightarrow 0 .
\end{aligned}
$$

It is a bit tricky but one can still prove the latter current $\tilde{\eta}_{1}^{\alpha \beta}$ to be zero, after explicitly writing out all its Lorentz indices. We have done this using the software Mathematica. 
[1] P. G. O. Freund and Y. Nambu, Dynamics in the ZweigIizuka Rule and a New Vector Meson Below 2-GeV/c ${ }^{2}$, Phys. Rev. Lett. 34, 1645 (1975).

[2] H. Fritzsch and P. Minkowski, Psi resonances, gluons and the Zweig rule, Nuovo Cimento A 30, 393 (1975).

[3] R. L. Jaffe and K. Johnson, Unconventional states of confined quarks and gluons, Phys. Lett. 60B, 201 (1976).

[4] A. Chodos, R. L. Jaffe, K. Johnson, C. B. Thorn, and V. F. Weisskopf, New extended model of hadrons, Phys. Rev. D 9, 3471 (1974).

[5] N. Isgur and J. E. Paton, Flux-tube model for hadrons in QCD, Phys. Rev. D 31, 2910 (1985).

[6] A. Szczepaniak, E. S. Swanson, C. R. Ji, and S. R. Cotanch, Glueball Spectroscopy in a Relativistic Many-Body Approach to Hadron Structure, Phys. Rev. Lett. 76, 2011 (1996).

[7] F. J. Llanes-Estrada, P. Bicudo, and S. R. Cotanch, $J^{--}$ Glueballs and a Low Odderon Intercept, Phys. Rev. Lett. 96, 081601 (2006).

[8] I. Szanyi, L. Jenkovszky, R. Schicker, and V. Svintozelskyi, Pomeron/glueball and odderon/oddball trajectories, Nucl. Phys. A998, 121728 (2020).

[9] L. Zhang, C. Chen, Y. Chen, and M. Huang, Spectra of glueballs and oddballs and the equation of state from holographic QCD, arXiv:2106.10748.

[10] K. G. Wilson, Confinement of quarks, Phys. Rev. D 10, 2445 (1974).

[11] Y. Chen et al., Glueball spectrum and matrix elements on anisotropic lattices, Phys. Rev. D 73, 014516 (2006).

[12] V. Mathieu, N. Kochelev, and V. Vento, The physics of glueballs, Int. J. Mod. Phys. E 18, 1 (2009).

[13] H. B. Meyer, Glueball regge trajectories, arXiv:hep-lat/ 0508002 .

[14] E. Gregory, A. Irving, B. Lucini, C. McNeile, A. Rago, C. Richards, and E. Rinaldi, Towards the glueball spectrum from unquenched lattice QCD, J. High Energy Phys. 10 (2012) 170.

[15] V. A. Novikov, M. A. Shifman, A. I. Vainshtein, and V. I. Zakharov, In search of scalar gluonium, Nucl. Phys. B165, 67 (1980).

[16] V. A. Novikov, M. A. Shifman, A. I. Vainshtein, and V. I. Zakharov, Are all hadrons alike?, Nucl. Phys. B191, 301 (1981).

[17] A. L. Kataev, N. V. Krasnikov, and A. A. Pivovarov, The connection between the scales of the gluon and quark worlds in perturbative QCD, Phys. Lett. 107B, 115 (1981).

[18] A. L. Kataev, N. V. Krasnikov, and A. A. Pivovarov, Two loop calculations for the propagators of gluonic currents, Nucl. Phys. B198, 508 (1982); Erratum, Nucl. Phys. B490, 505 (1997).

[19] S. Narison, Spectral function sum rules for gluonic currents, Z. Phys. C 26, 209 (1984).

[20] S. Narison, Masses, decays and mixings of gluonia in QCD, Nucl. Phys. B509, 312 (1998).

[21] E. Bagan and T. G. Steele, Mass of the scalar glueball. Higher-loop effects in the QCD sum rules, Phys. Lett. B 243, 413 (1990).

[22] J. I. Latorre, S. Narison, and S. Paban, $0^{++}$trigluonium sum rules, Phys. Lett. B 191, 437 (1987).
[23] H. Forkel, Direct instantons, topological charge screening and QCD glueball sum rules, Phys. Rev. D 71, 054008 (2005).

[24] G. Hao, C. F. Qiao, and A. L. Zhang, $0^{-+}$trigluon glueball and its implication for a recent BES observation, Phys. Lett. B 642, 53 (2006).

[25] C. F. Qiao and L. Tang, Finding the $0^{--}$Glueball, Phys. Rev. Lett. 113, 221601 (2014).

[26] L. Tang and C. F. Qiao, Mass spectra of $0^{+-}, 1^{-+}$, and $2^{+-}$ exotic glueballs, Nucl. Phys. B904, 282 (2016).

[27] A. Pimikov, H. J. Lee, N. Kochelev, and P. Zhang, Is the exotic $0^{--}$glueball a pure gluon state? Phys. Rev. D 95, 071501(R) (2017).

[28] A. Pimikov, H. J. Lee, and N. Kochelev, Comment on "Finding the $0^{--}$Glueball", Phys. Rev. Lett. 119, 079101 (2017).

[29] A. Pimikov, H. J. Lee, N. Kochelev, P. Zhang, and V. Khandramai, Exotic glueball $0^{ \pm-}$states in QCD sum rules, Phys. Rev. D 96, 114024 (2017).

[30] S. Narison, Di-gluonium sum rules, $I=0$ scalar mesons and conformal anomaly, Nucl. Phys. A1017, 122337 (2022).

[31] V. M. Abazov et al. (D0 Collaboration), Measurement of the differential cross section $d \sigma / d t$ in elastic $p \bar{p}$ scattering at $\sqrt{s}=1.96 \mathrm{TeV}$, Phys. Rev. D 86, 012009 (2012).

[32] V. M. Abazov et al. (D0 and TOTEM Collaborations), Comparison of $p p$ and $p \bar{p}$ Differential Elastic Cross Sections and Observation of the Exchange of a Colorless C-Odd Gluonic Compound, Phys. Rev. Lett. 127, 062003 (2021).

[33] G. Antchev et al. (TOTEM Collaboration), First determination of the $\rho$ parameter at $\sqrt{s}=13 \mathrm{TeV}$ : Probing the existence of a colourless $C$-odd three-gluon compound state, Eur. Phys. J. C 79, 785 (2019).

[34] E. M. Levin and M. G. Ryskin, High-energy hadron collisions in QCD, Phys. Rep. 189, 268 (1990).

[35] M. A. Braun, Odderon and QCD, arXiv:hep-ph/9805394.

[36] J. R. Cudell, V. V. Ezhela, P. Gauron, K. Kang, Yu. V. Kuyanov, S. B. Lugovsky, E. Martynov, B. Nicolescu, E. A. Razuvaev, and N. P. Tkachenko (COMPETE Collaboration), Benchmarks for the Forward Observables at RHIC, the Tevatron-Run II and the LHC, Phys. Rev. Lett. 89, 201801 (2002).

[37] V. A. Khoze, A. D. Martin, and M. G. Ryskin, Elastic proton-proton scattering at $13 \mathrm{TeV}$, Phys. Rev. D 97, 034019 (2018).

[38] E. Martynov and B. Nicolescu, Odderon effects in the differential cross-sections at Tevatron and LHC energies, Eur. Phys. J. C 79, 461 (2019).

[39] V. A. Khoze, A. D. Martin, and M. G. Ryskin, Black disk, maximal Odderon and unitarity, Phys. Lett. B 780, 352 (2018).

[40] T. Csörgő, R. Pasechnik, and A. Ster, Odderon and proton substructure from a model-independent Lévy imaging of elastic $p p$ and $p \bar{p}$ collisions, Eur. Phys. J. C 79, 62 (2019).

[41] V. P. Gonçalves and P. V. R. G. Silva, The Phillips-Barger model for the elastic cross section and the Odderon, Eur. Phys. J. C 79, 237 (2019).

[42] W. Xie, A. Watanabe, and M. Huang, Elastic proton-proton scattering at LHC energies in holographic QCD, J. High Energy Phys. 10 (2019) 053. 
[43] L. Lukaszuk and B. Nicolescu, A possible interpretation of $p p$ rising total cross-sections, Lett. Nuovo Cim. 8, 405 (1973).

[44] J. Bartels, High-energy behavior in a nonabelian gauge theory (II). First corrections to $T_{n \rightarrow m}$ beyond the leading $\ln s$ approximation, Nucl. Phys. B175, 365 (1980).

[45] J. Kwiecinski and M. Praszalowicz, Three gluon integral equation and odd $\mathrm{C}$ singlet Regge singularities in QCD, Phys. Lett. 94B, 413 (1980).

[46] A. Donnachie and P. V. Landshoff, Multi-gluon exchange in pp elastic scattering, Phys. Lett. 123B, 345 (1983).

[47] A. Dumitru and R. Paatelainen, Cubic color charge correlator in a proton made of three quarks and a gluon, arXiv:2106.12623.

[48] M. M. Block, Hadronic forward scattering: Predictions for the Large Hadron Collider and cosmic rays, Phys. Rep. 436, 71 (2006).

[49] R. L. Jaffe, K. Johnson, and Z. Ryzak, Qualitative features of the glueball spectrum, Ann. Phys. (N.Y.) 168, 344 (1986).

[50] H. X. Chen, W. Chen, and S. L. Zhu, Toward the existence of the odderon as a three-gluon bound state, Phys. Rev. D 103, L091503 (2021).

[51] C. N. Yang, Selection rules for the dematerialization of a particle into two photons, Phys. Rev. 77, 242 (1950).
[52] M. A. Shifman, A. I. Vainshtein, and V. I. Zakharov, QCD and resonance physics. Theoretical foundations, Nucl. Phys. B147, 385 (1979).

[53] L. J. Reinders, H. Rubinstein, and S. Yazaki, Hadron properties from QCD sum rules, Phys. Rep. 127, 1 (1985).

[54] H. X. Chen, A. Hosaka, and S. L. Zhu, Light scalar tetraquark mesons in the QCD sum rule, Phys. Rev. D 76, 094025 (2007).

[55] H. X. Chen, E. L. Cui, W. Chen, X. Liu, T. G. Steele, and S. L. Zhu, QCD sum rule study of hidden-charm pentaquarks, Eur. Phys. J. C 76, 572 (2016).

[56] W. Chen, H. X. Chen, X. Liu, T. G. Steele, and S. L. Zhu, Hunting for exotic doubly hidden-charm/bottom tetraquark states, Phys. Lett. B 773, 247 (2017).

[57] J. Govaerts, F. de Viron, D. Gusbin, and J. Weyers, QCD Sum rules and hybrid mesons, Nucl. Phys. B248, 1 (1984).

[58] S. Narison, QCD parameter correlations from heavy quarkonia, Int. J. Mod. Phys. A 33, 1850045 (2018); 33, 1892004(A) (2018).

[59] S. Narison, Gluon condensates and precise $\bar{m}_{c, b}$ from QCDmoments and their ratios to order $\alpha_{s}^{3}$ and $\left\langle G^{4}\right\rangle$, Phys. Lett. B 706, 412 (2012).

[60] P. A. Zyla et al. (Particle Data Group), Review of particle physics, Prog. Theor. Exp. Phys. 2020, 083 C01 (2020). 